\title{
Explainable Deep Learning-Assisted Fluorescence Discrimination for Aminoglycoside Antibiotic Identification
}

\author{
Xiaoqing Tan ${ }^{\mathrm{a}}$, Yongpeng Liang ${ }^{\mathrm{a}}$, Yingying Ye ${ }^{\mathrm{a}}$, Zhihao Liu ${ }^{\mathrm{a}}$, Jianxin Meng ${ }^{\mathrm{a}}$ and \\ Fengyu Li*ab \\ ${ }^{a}$ College of Chemistry and Materials Science, Guangdong Provincial Key Laboratory of Functional \\ Supramolecular Coordination Materials and Applications, Guangdong Engineering \& Technology \\ Research Centre of Graphene-like Materials and Products, Jinan University, Guangzhou 510632, China \\ ${ }^{b}$ College of Chemistry, Zhengzhou University, Zhengzhou 450001, China.
}

E-mail: $\underline{\text { lifengyu@jnu.edu.cn }}$ 


\section{Table of Contents}

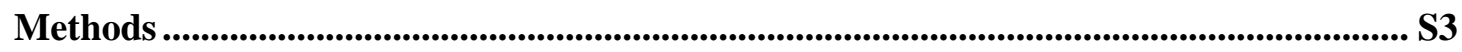

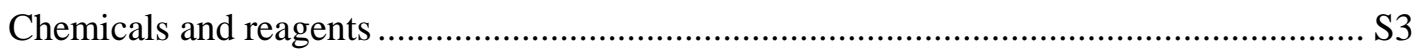

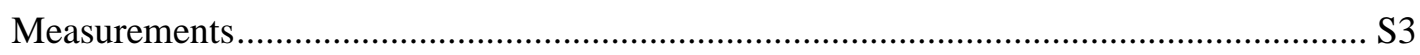

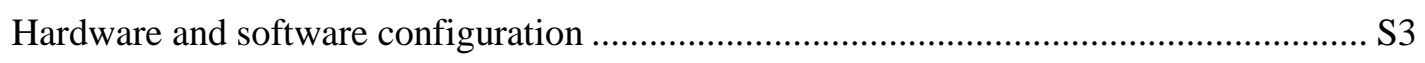

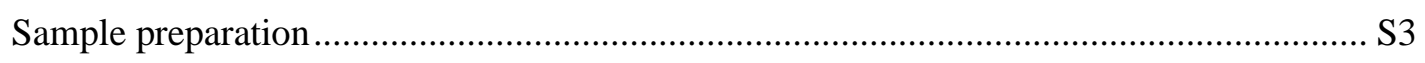

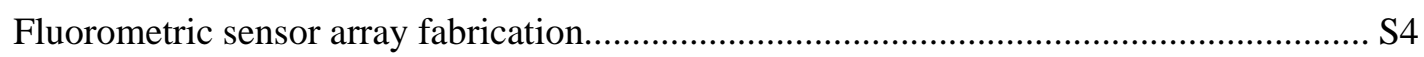

Images collecting and processing for modeling ............................................................ S4

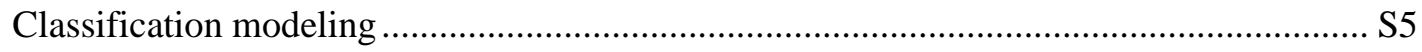

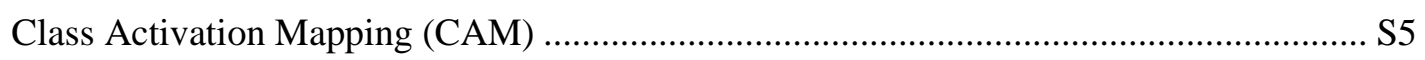

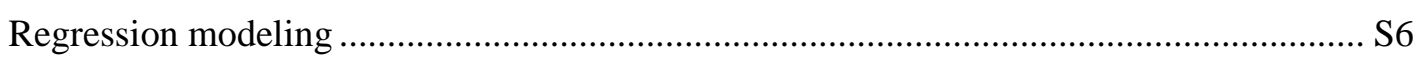

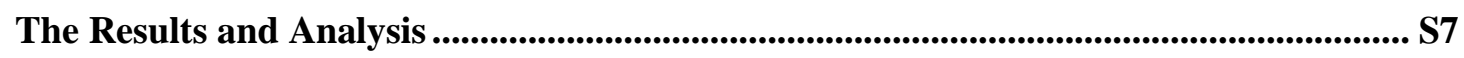

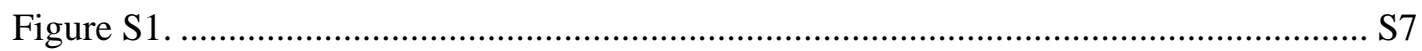

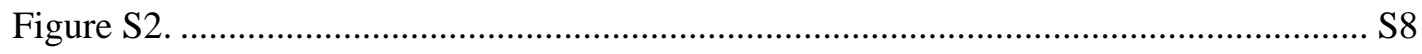

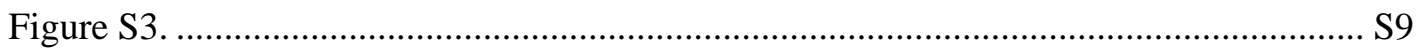

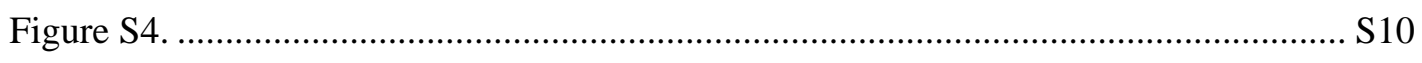

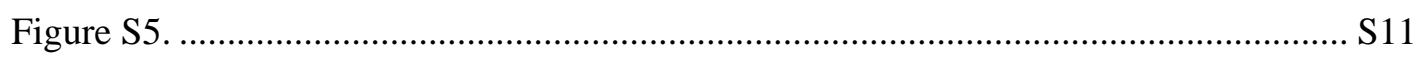

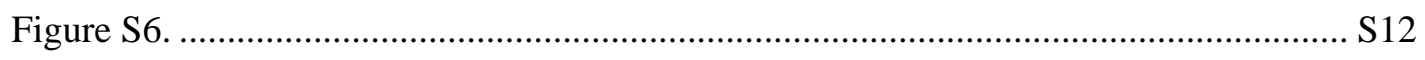

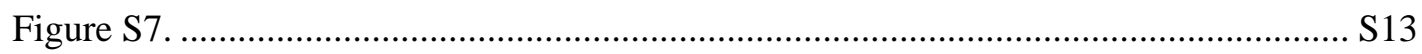

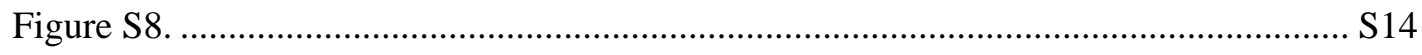

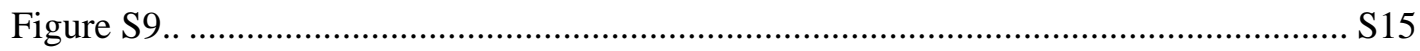

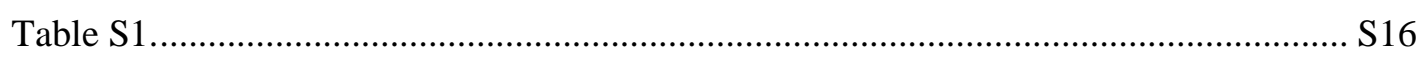

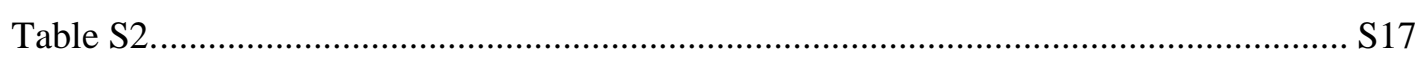

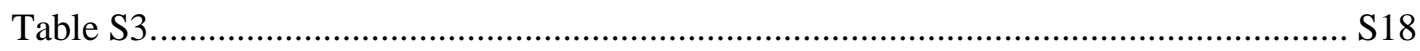

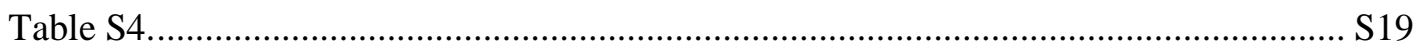

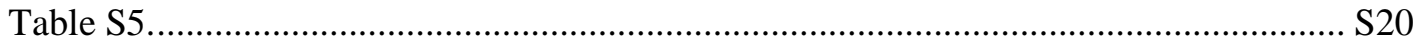

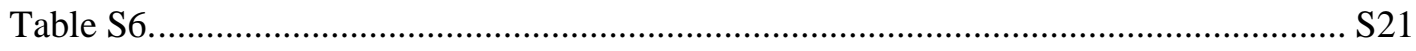

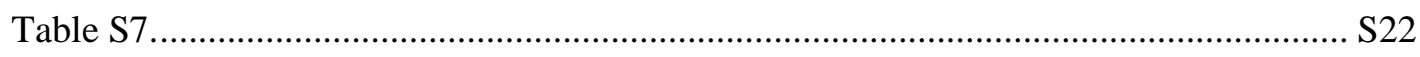

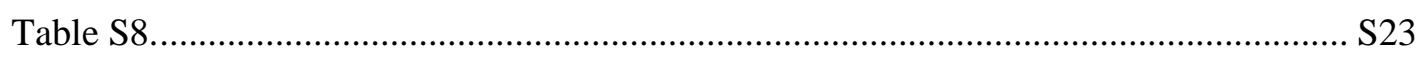




\section{Methods}

\section{Chemicals and reagents}

2, 3- Naphthalenedicarboxaldehyde (NDA, 99\%), Diphenylborinic acid 2-aminoethyl ester (DPBA, 98\%), Kanamycin sulfate (Kan, >94\%), Gentamycin sulfate (Gen, 59\%), Tobramycin (Tob, 98\%), Amikacin sulfate (Amk, >95\%), 1,4-Diaminobutane (Putrescine, 98\%), Urea (99\%), Phosphate buffered saline (PBS, 0.01M, pH 7.4) were all purchased from Shanghai Macklin Biochemical Co., Ltd. Streptomycin sulfate (Strep, >95\%), Neomycin sulfate (Neo, $>97 \%$ ) were all purchased from TCI (Shanghai) Chemical Industrial Development Co., Ltd. Alizarin red-S (ARS, $\geq 80 \%$ ), Sucrose (AR) were purchased from Shanghai Aladdin Biochemical Technology Co., Ltd. Sulforhodamine B (SRB, 95\%) were purchased from Shanghai Jinsui Biotechnology Co., Ltd. $\mathrm{NaHSO}_{3}$ (AR) were purchased from Shanghai Eon Chemical Technology Co., Ltd. L-Glutamic acid (99\%), Tyramine (97\%) were purchased from Beijing Balinwei Technology Co., Ltd. Soluble starch (AR) were purchased from Guangzhou Chemical Reagent Factory. Corning 384-well flat clear bottom black polystyrene TC-treated microplates were purchased from Corning Incorporated.

\section{Measurements}

Fluorescence spectra were measured by a Shimadzu RF-5301PC spectrofluorophotometer. $\begin{array}{lllll}\text { Fluorescent images were recorded using ChampChemi } & \\ & & \end{array}$ Chemiluminescent/Fluorescent/Gel Imaging and Analysis System.

\section{Hardware and software configuration}

All the computation and learning methods were complemented in Visual Studio Code (version 1.55.0) in Microsoft Windows 10 (x64). The CPU is Intel Core i5-8265U and the memory is $8 \mathrm{~GB}$. The construction of DL models is not accelerated by GPU. All deep learning models (MLP, CNN) were constructed by PyTorch. All machine learning models were constructed by scikit-learn. The XGBoost model was constructed by XGBoost library.

\section{Sample preparation}

A series of AGs solutions of different categories (Amk, Kan, Strep, Gen, Tob, Neo) and concentrations (10 mg/L $100 \mathrm{mg} / \mathrm{L}$, with a concentration taken at intervals of $5 \mathrm{mg} / \mathrm{L})$ with 
different aqueous solutions as solvents (Table S1) were prepared. In addition, 312 blank samples (i.e. $0 \mathrm{mg} / \mathrm{L}$ ) were prepared using different aqueous solutions as solvents without AGs as controls.

\section{Fluorometric sensor array fabrication}

The preparation scheme of the three sensing element solutions is as follows. For the NDA sensing element, the mixed solution of NDA ( $400 \mu \mathrm{M}$, NDA solids are initially dissolved in anhydrous ethanol) and $\mathrm{NaHSO}_{3}(400 \mu \mathrm{M})$ was prepared by using PBS as solvent. For the ARSDPBA sensing element, the mixed solution of ARS $(20 \mu \mathrm{M})$ and DPBA $(200 \mu \mathrm{M})$ was prepared by using PBS as solvent. For the SRB sensing element, SRB $(0.2 \mu \mathrm{M})$ solution is prepared with ultrapure water.

The array experiment for qualitative and quantitative of AGs was performed in 384-well microplates. The fluids (NDA sensing element solutions, ARS-DPBA sensing element solutions, SRB sensing element solutions, and the prepared analyte solutions), were added with a multi-channel pipette as follows. Each of 3 wells was added with $55 \mu \mathrm{L}$ of the 3 sensing element solutions. Subsequently, $55 \mu \mathrm{L}$ of analyte solutions or blank solutions were dispensed. After this period, the plate was ultrasonic shaken for $1 \mathrm{~min}$. Timing started when the analyte solutions were added to the microplates, and images collection can be carried out after 20 mins response.

\section{Images collecting and processing for modeling}

Fluorescence intensity images of the sensor array were recorded in 6 channels $(\mathrm{CH} 1$ : ex $=470 \mathrm{~nm}$ em $=505 \mathrm{~nm}, \mathrm{CH} 2:$ ex $=470 \mathrm{~nm}$ em $=535 \mathrm{~nm}, \mathrm{CH} 3:$ ex $=470 \mathrm{~nm}$ em $=570 \mathrm{~nm}, \mathrm{CH} 4:$ ex $=470 \mathrm{~nm}$ em $=590 \mathrm{~nm}, \mathrm{CH} 5:$ ex $=530 \mathrm{~nm}$ em $=570 \mathrm{~nm}$, CH6: ex=530nm em=590nm) by the chemiluminescent imaging system. For an independent sample, 6 images taken under 6 channels are automatically cropped and stitched together by the program to form a sample image. The program is written in Python language. Python Image Library, a python image processing library, was mainly used.

When modeling using MLP and ML algorithms, the input data must be a two-dimensional matrix. Because the size of each image is $240 \times 120$, if simply unfolding an image into a long sequence of numbers, a matrix with 28800 feature dimensions and 8496 samples will be obtained, facing the curse of dimensionality. As a result, the model will be prone to over-fitting and even fail to converge if the feature dimensions greatly exceed the number of samples in the input data. Therefore, another program was written to transform the high-dimensional picture 
data into a two-dimensional matrix with only 18 feature dimensions. For each sample image, the program read the pixel values of 18 squares that represent the corresponding FI in each sample image respectively. Then the pixel values were added up and a vector containing 18 elements was obtained. For a data set with $\mathrm{n}$ samples, a two-dimensional matrix with the size of $n \times 18$ is finally generated to be used as the total data set for modeling. The program is written in Python language. OpenCV library was used to read the pixel value of the picture, and Numpy library was used to process the pixel value into a matrix.

\section{Classification modeling}

A total of unduplicated 8,496 fluorescence intensity images for the 6 kinds of AGs were obtained as the dataset, where each AGs category has 1,416 images. The total dataset was divided into three subsets: training set, validation set and external unlabeled test set, at a ratio of $8: 1: 1$

The architecture of the CNN classification model is summarized in Table S2. During the training and testing, each image was resized to $100 \times 100$ pixels as input. The loss function was defined as cross entropy, and adaptive moment estimation (Adam) was chosen for stochastic optimization. The loss (cross entropy) and accuracy of the model were used to evaluate the model. The learning rate was set to 0.0001 , the batch size of training set and validation set was 128. In order to improve the generalization ability of the model, the early stopping method was used in the training process to prevent overfitting. This method monitored the performance of the model on the validation set during training. When the performance of the model on the validation set begins to decline (we set the loss of validation set not to drop after 20 epochs), the training will be stopped. As a result, 167 epochs were totally trained.

The architecture of the MLP classification model is summarized in Table S3. The optimization and evaluation methods of the model are the same as those of CNN classification model. The learning rate was set to 0.0005 , the batch size of training set and validation set was 128 , and 512 epochs were totally trained.

The parameters of ML classification models are summarized in Table S4. In the DT model, the importance of a feature was computed as the (normalized) total reduction of the criterion brought by that feature. It is also known as the Gini importance. The importance of 18 features can be obtained by scikit-learn.

\section{Class Activation Mapping (CAM)}


Class activation maps (CAMs) were used to visualize which parts of the fluorescence intensity images have importance for AGs classification. The procedure for generating CAMs is to use a global average pooling (GAP) layer at the end of neural networks instead of a fullyconnected layer (Figure S9). The key of the procedure is to get the product of weights and feature maps of the last layer. The feature maps can be extracted using the Hook function in PyTorch. The weights are obtained by taking out the parameter list of the network, and then the corresponding last pair is the weights and bias of the last fully-connected layer. The weights and feature maps are multiplied into matrix which represents the class activation map. By simply up-sampling the class activation map to the size of the input image, the image regions most relevant to the particular category can be identified. In order to comprehensively demonstrate how the CNN model utilizes the features of the fluorescence intensity images, the CAMs of all samples was obtained. Then we average the CAMs for each category to produce a visualization that contains more comprehensive details and thus is able to more accurately represent what regions of the fluorescence intensity images are most important for the model when making a specific prediction.

\section{Regression modeling}

The initial classification modeling data sets were split into 6 datasets according to the AGs category, and each dataset contains 312 blank samples and 1416 corresponding samples in different concentrations $(10 \mathrm{mg} / \mathrm{L} \sim 100 \mathrm{mg} / \mathrm{L}$, with a concentration taken at intervals of 5 $\mathrm{mg} / \mathrm{L}$ ) and different solution environments (Table S1). Each dataset was divided into three subsets, training set, validation set and test set, at a ratio of $8: 1: 1$.

The architecture of the CNN regression model is summarized in Table S5. The pixels size of the input image was resized to $100 \times 100$. The loss function was defined as MSE, and Adam was chosen for stochastic optimization. The quality of the trained models was evaluated by four evaluation metrics: $\mathrm{R}^{2}$, MSE, RMSE, MAE. Learning rates of regression models corresponding to the 6 AGs were all set to 0.0005 , the batch size of training set and validation set was 32 . The early stopping method was also adopted in the training process, and different AGs regression models required different numbers of training epochs.

The architecture of the MLP regression model is summarized in Table S6. The optimization and evaluation methods of the model are the same as those of CNN regression model. The learning rate was set to 0.001 , the batch size of training set and validation set was 256 , and 512 epochs were totally trained. The parameters of ML regression models are summarized in Table S7. 


\section{The Results and Analysis}

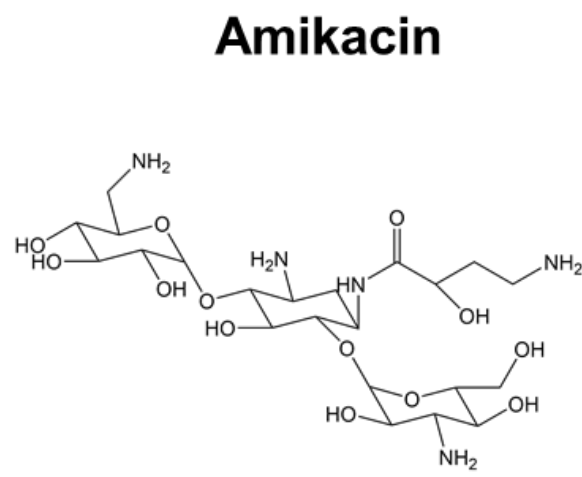

Streptomycin

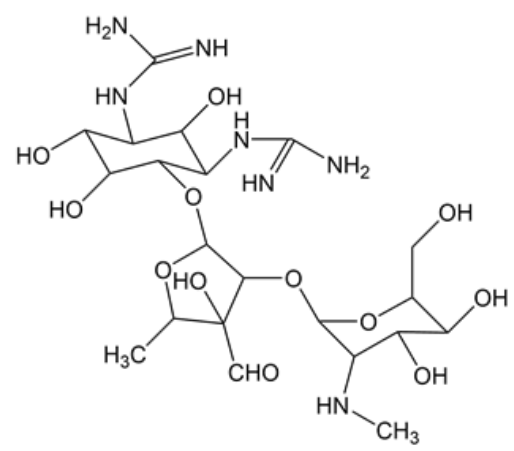

Tobramycin

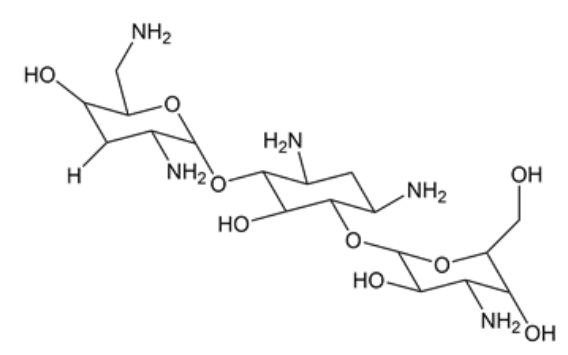

Kanamycin

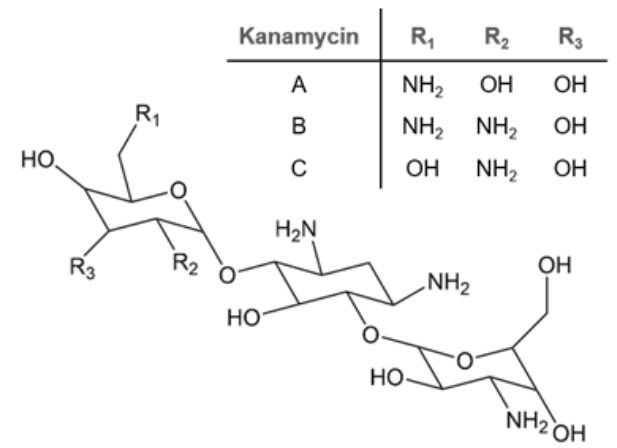

Gentamycin

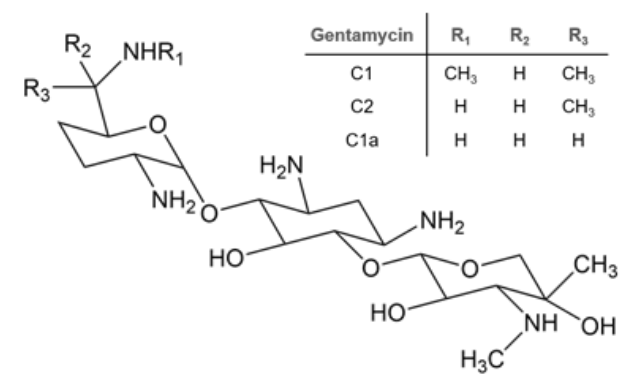

Neomycin

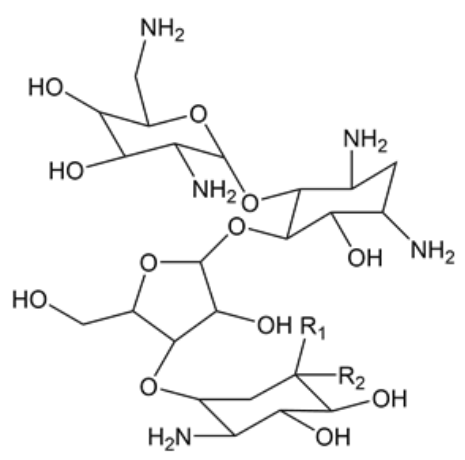

Figure S1. The chemical structure of 6 different categories of AGs. 

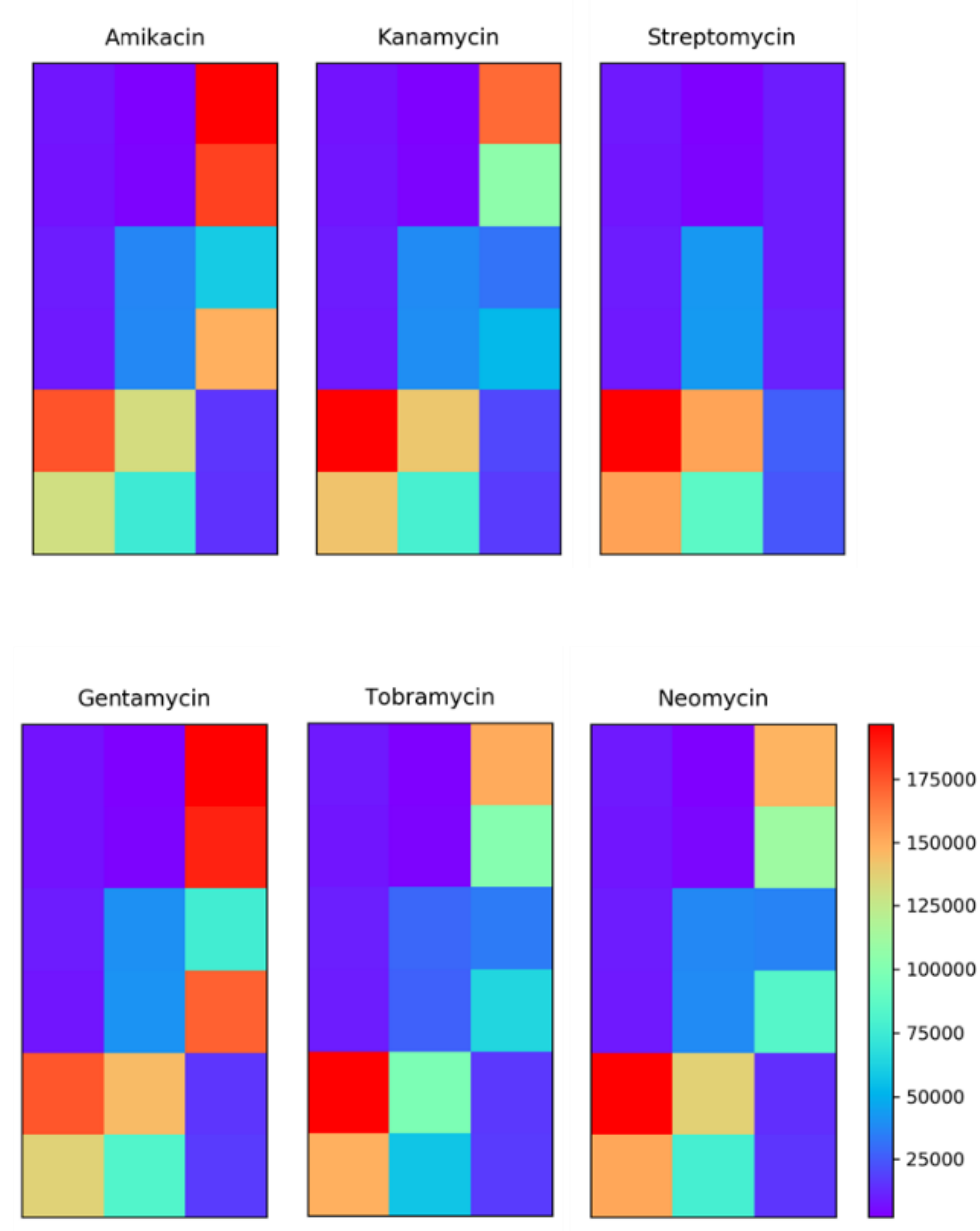

Figure S2. Heat maps of the fluorescence response patterns of 6 AGs $(100 \mathrm{mg} / \mathrm{L}, 24$ duplicates). 


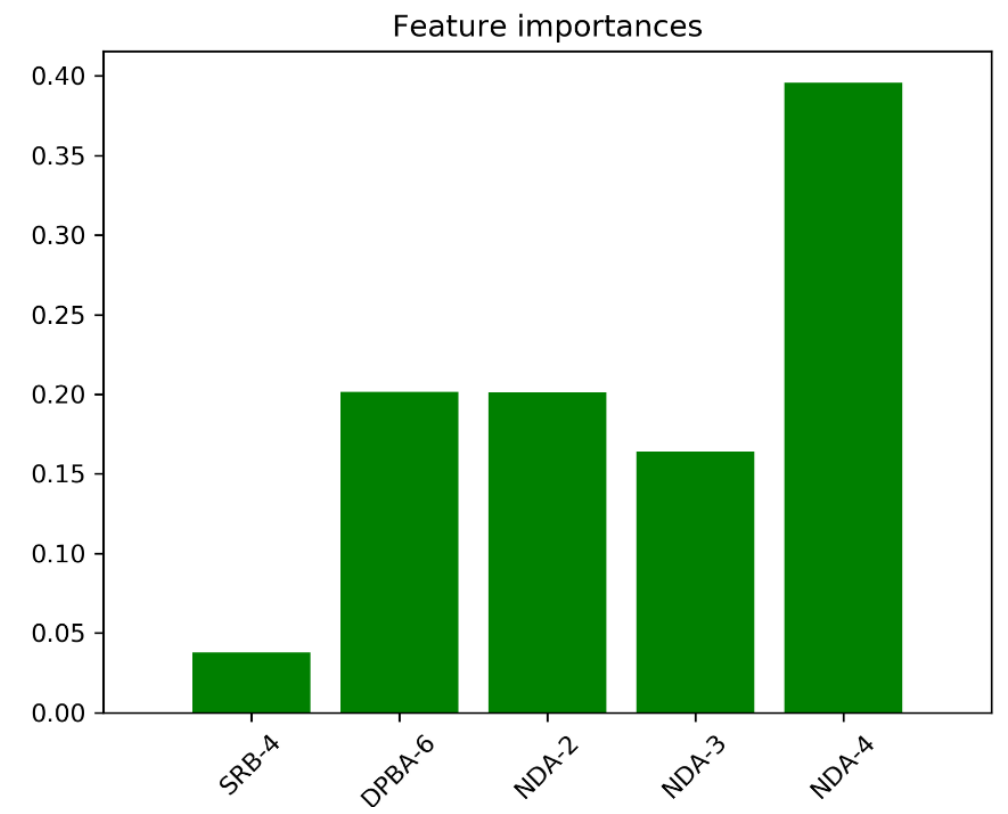

Figure S3. Feature importance result graph of classification of 6 AGs $(100 \mathrm{mg} / \mathrm{L}, 24$ duplicates) based on DT model with 5 most important features. 


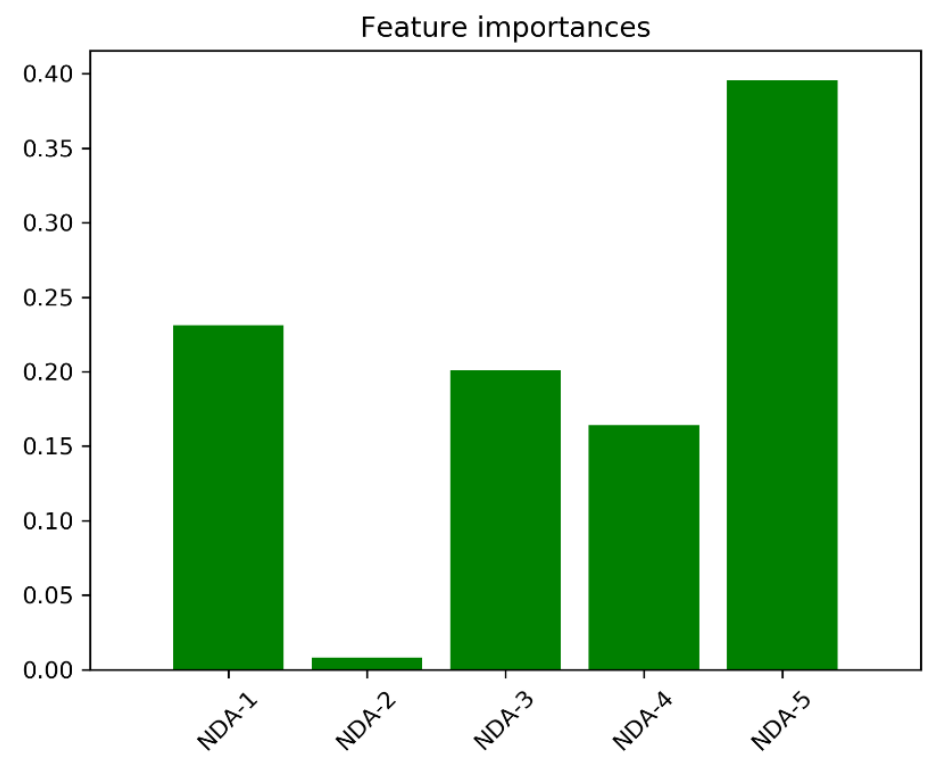

Figure S4. Feature importance result graph of classification of 6 AGs $(10 \mathrm{mg} / \mathrm{L} \sim 100 \mathrm{mg} / \mathrm{L}$, in different solution environment) based on DT model with 5 most important features. 

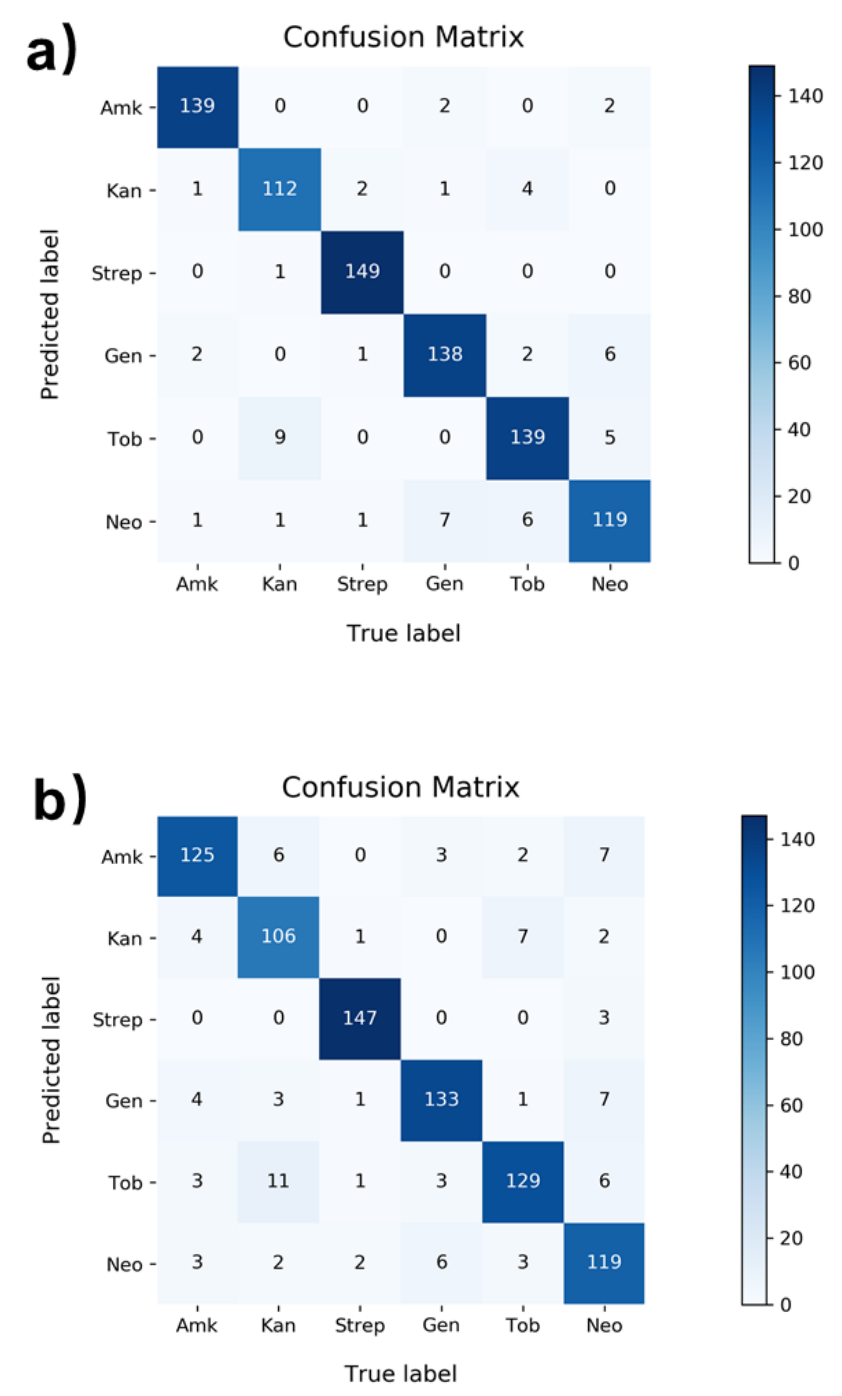

Figure S5. Confusion matrixes of the DT prediction for the test set a) before and b) after feature extraction process. 


\begin{tabular}{|c|c|c|c|c|c|c|c|}
\hline \multirow{2}{*}{\multicolumn{2}{|c|}{$\begin{array}{l}\text { Training set } \\
\text { Confusion matrix }\end{array}$}} & \multicolumn{6}{|c|}{ True label } \\
\hline & & Amk & Kan & Strep & Gen & Tob & $\mathrm{Neo}$ \\
\hline \multirow{6}{*}{ 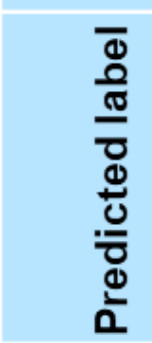 } & Amk & 1138 & 0 & 0 & 0 & 0 & 0 \\
\hline & Kan & 0 & 1129 & 0 & 0 & 0 & 0 \\
\hline & Strep & 0 & 0 & 1142 & 0 & 0 & 0 \\
\hline & Gen & 0 & 0 & 0 & 1126 & 0 & 0 \\
\hline & Tob & 0 & 0 & 0 & 0 & 1115 & 0 \\
\hline & $\mathrm{Neo}$ & 0 & 0 & 0 & 0 & 0 & 1146 \\
\hline
\end{tabular}

Validation set

True label

Confusion matrix

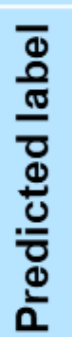

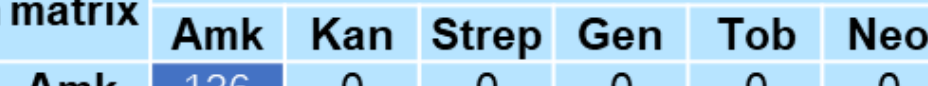

\begin{tabular}{|c|c|c|c|c|c|c|c|} 
Amk & 136 & 0 & 0 & 0 & 0 & 0 \\
\hline
\end{tabular}

\begin{tabular}{l|c|c|c|c|c|c|} 
Kan & 0 & 153 & 0 & 0 & 0 & 0
\end{tabular}

\begin{tabular}{|l|l|l|l|l|l|l|} 
Strep & 0 & 0 & 142 & 0 & 0 & 0
\end{tabular}

\begin{tabular}{l|l|l|l|l|l|l|} 
Gen & 0 & 0 & 0 & 135 & 0 & 0
\end{tabular}

\begin{tabular}{c|c|c|c|c|c|c|} 
Tob & 0 & 0 & 0 & 0 & 146 & 0 \\
\hline
\end{tabular}

\begin{tabular}{l|l|l|l|l|l|l|} 
Neo & 0 & 0 & 0 & 0 & 0 & 138 \\
\hline
\end{tabular}

Test set

Confusion matrix

Amk

Amk Kan

True label

\begin{tabular}{|c|c|c|c|c|c|c|c|}
\hline & Amk & 142 & 0 & 0 & 0 & 0 & 0 \\
\hline & Kan & 0 & 134 & 0 & 0 & 0 & 0 \\
\hline & Strep & 0 & 0 & 132 & 0 & 0 & 0 \\
\hline & Gen & 0 & 0 & 0 & 153 & 0 & 0 \\
\hline & Tob & 0 & 0 & 0 & 0 & 155 & 0 \\
\hline & $\mathrm{Neo}$ & 0 & 0 & 0 & 2 & 0 & 132 \\
\hline
\end{tabular}

Figure S6. Confusion matrixes of the CNN prediction for the training set, validation set and test set after feature extraction process. 

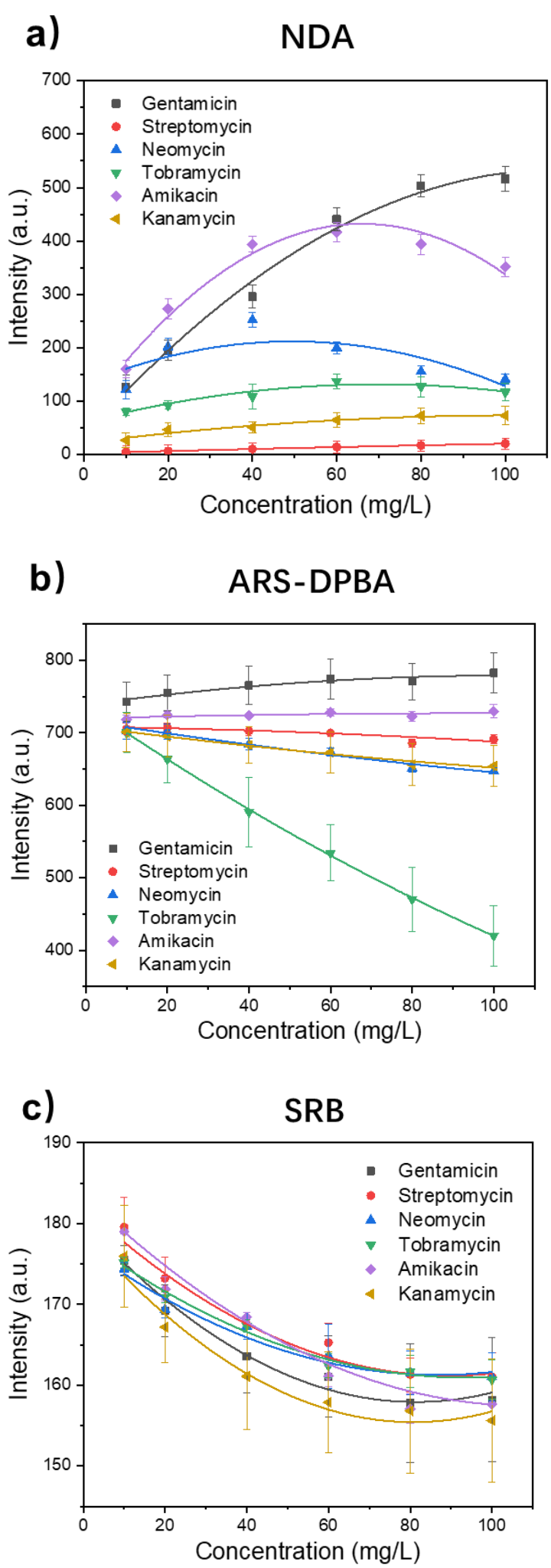

Figure S7. Concentration-fluorescence spectral intensity curve of AGs in a) NDA, b) ARSDPBA, c) SRB sensing system. 

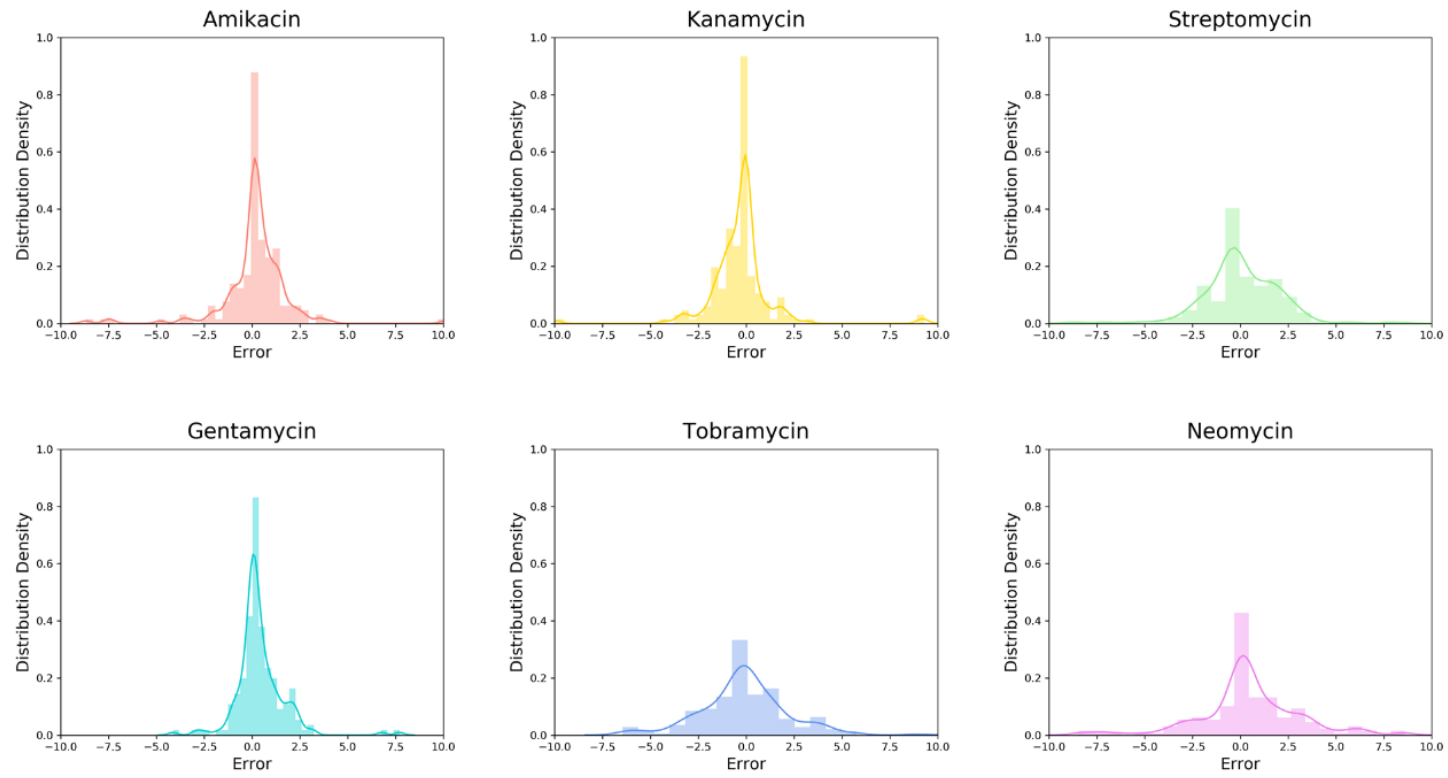

Figure S8. The prediction error distribution for 6 AGs on the test set. 


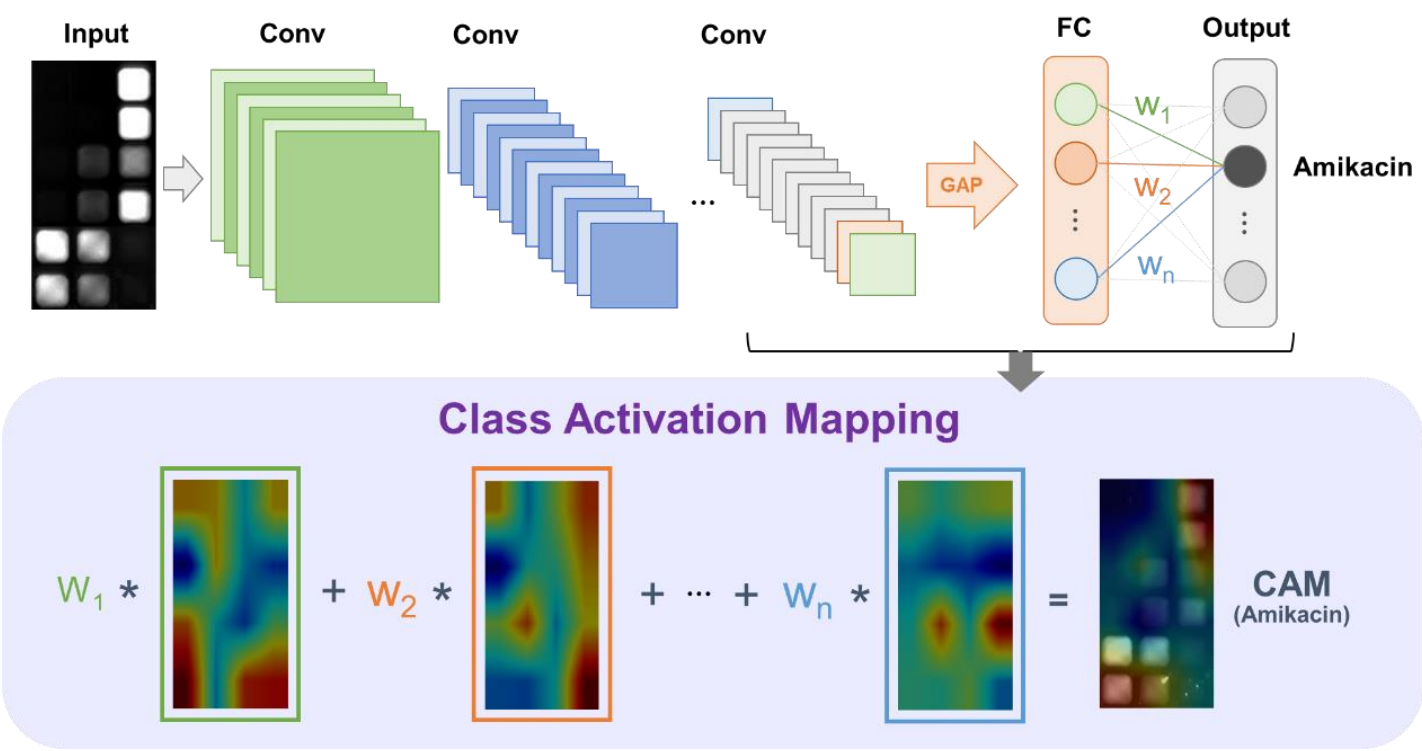

Figure S9. Class Activation Mapping: the predicted class score is mapped back to the previous convolutional layer to generate the class activation maps (CAMs). The CAM highlights the class-specific discriminative regions. 
Table S1. Solvent composition for preparing AGs solution.

\begin{tabular}{|c|c|c|}
\hline NO. & Type & Source \\
\hline 1 & Ultrapure water & $\begin{array}{l}\text { Prepared using YL-100BD (V5) laboratory ultrapure } \\
\text { water machine (EREE, China) and used throughout. }\end{array}$ \\
\hline 2 & \multirow{3}{*}{$\begin{array}{l}\text { Disturbing component } \\
\text { solution }\end{array}$} & $\begin{array}{l}\text { L-Glutamic acid, soluble starch, sucrose (each } \\
\text { component has a concentration of } 100 \mathrm{mg} / \mathrm{L} \text { ). }\end{array}$ \\
\hline 3 & & $\begin{array}{l}\text { Tyramine, urea, glutamic acid, sucrose (each component } \\
\text { has a concentration of } 100 \mathrm{mg} / \mathrm{L} \text { ). }\end{array}$ \\
\hline 4 & & $\begin{array}{l}\text { Putrescine, tyramine, urea, L-Glutamic acid, soluble } \\
\text { starch, sucrose (each component has a concentration of } \\
100 \mathrm{mg} / \mathrm{L} \text { ). }\end{array}$ \\
\hline 5 & \multirow{6}{*}{$\begin{array}{l}\text { Actual water samples from } \\
\text { different places }\end{array}$} & $\begin{array}{l}\text { The Pearl River (located at } 23^{\circ} 6^{\prime} 40^{\prime \prime} \text { north latitude and } \\
113^{\circ} 19^{\prime} 55^{\prime \prime} \text { east longitude of Guangzhou City, } \\
\text { Guangdong Province). }\end{array}$ \\
\hline 6 & & $\begin{array}{l}\text { Domestic sewage in the student living area of Jinan } \\
\text { University (located at } 23^{\circ} 7^{\prime} 53^{\prime \prime} \text { north latitude and } \\
113^{\circ} 20^{\prime} 53^{\prime \prime} \text { east longitude of Guangzhou City, } \\
\text { Guangdong Province). }\end{array}$ \\
\hline 7 & & $\begin{array}{l}\text { The Jing Lake (located at } 23^{\circ} 1^{\prime} 0^{\prime \prime} \text { north latitude and } \\
113^{\circ} 24^{\prime} 54^{\prime \prime} \text { east longitude of Guangzhou City, } \\
\text { Guangdong Province) }\end{array}$ \\
\hline 8 & & $\begin{array}{l}\text { Wastewater from Guangdong Maternal and Child Health } \\
\text { Hospital (located at } 23^{\circ} 8^{\prime} 53^{\prime \prime} \text { north latitude and } \\
113^{\circ} 14^{\prime} 54^{\prime \prime} \text { east longitude of Guangzhou City, } \\
\text { Guangdong Province). }\end{array}$ \\
\hline 9 & & $\begin{array}{l}\text { Water from a duck farm pond (located at } 23^{\circ} 1^{\prime} 20^{\prime \prime} \text { north } \\
\text { latitude and } 113^{\circ} 25^{\prime} 15^{\prime \prime} \text { east longitude of Guangzhou } \\
\text { City, Guangdong Province). }\end{array}$ \\
\hline 10 & & $\begin{array}{l}\text { Water from a fishpond (located at } 23^{\circ} 1^{\prime} 18^{\prime \prime} \text { north latitude } \\
\text { and } 113^{\circ} 25^{\prime} 18^{\prime \prime} \text { east longitude of Guangzhou City, } \\
\text { Guangdong Province). }\end{array}$ \\
\hline
\end{tabular}


Table S2. The architecture of the CNN classification model.

\begin{tabular}{|c|c|c|}
\hline Layer & Layer (type) & Output Shape \\
\hline 1 & Input layer & (None, $100,100,3$ ) \\
\hline 2 & Convolution layer & (None, 98, 98, 32) \\
\hline 3 & Max pooling layer & (None, 49, 49, 32) \\
\hline 4 & Convolution layer & (None, 47, 47, 64) \\
\hline 5 & Max pooling layer & (None, 23, 23, 64) \\
\hline 6 & Convolution layer & (None, $21,21,128)$ \\
\hline 7 & Max pooling layer & (None, $10,10,128$ ) \\
\hline 8 & Convolution layer & (None, $8,8,128$ ) \\
\hline 9 & Max pooling layer & (None, $4,4,128$ ) \\
\hline 10 & Flatten layer & (None, 2048) \\
\hline 11 & Fully-connected layer & (None, 128) \\
\hline 12 & Dropout layer & (None, 128) \\
\hline 13 & Fully-connected layer & (None, 6) \\
\hline 14 & Output & (None, 6) \\
\hline
\end{tabular}


Table S3. The architecture of the MLP classification model.

\begin{tabular}{|c|c|c|}
\hline Layer & Layer (type) & Output Shape \\
\hline 1 & Input layer & (None, 18) \\
\hline 2 & Fully-connected layer & (None, 64) \\
\hline 3 & Fully-connected layer 256$)$ \\
\hline 4 & Fully-connected layer & (None, 128) \\
\hline 5 & Dropout layer & (None, 128) \\
\hline 6 & Fully-connected layer & (None, 6) \\
\hline 7 & Output & (None, 6) \\
\hline
\end{tabular}


Table S4. Parameters of ML classification models.

\begin{tabular}{|c|c|}
\hline Algorithm & Parameters \\
\hline $\begin{array}{l}\text { Logistic } \\
\text { Regression }\end{array}$ & $($ solver $=$ 'sag', penalty $=$ '12', C $=14$, max_iter $=560)$ \\
\hline Decision trees & $\begin{array}{l}(\text { criterion }=\text { 'gini', splitter }=\text { 'best', max_depth }=21, \text { min_samples_leaf }=1 \text {, } \\
\text { min_samples_split }=2)\end{array}$ \\
\hline Random forest & $\begin{array}{l}(\text { criterion }=\text { 'gini', } \mathrm{n} \text { _estimators }=41, \text { max_depth }=17, \text { min_samples_leaf }=1 \text {, } \\
\text { min_samples_split }=2)\end{array}$ \\
\hline SVM & $($ kernel $=$ 'rbf', gamma $=1.68, \mathrm{C}=2.46)$ \\
\hline KNN & $\begin{array}{l}\left(\mathrm{n} \_ \text {neighbors }=1, \text { weights }=\text { 'uniform', algorithm }=\text { 'auto', leaf_size }=30, p=2 \text {, }\right. \\
\text { metric }=\text { 'minkowski') }\end{array}$ \\
\hline XGBoost & $($ num_round $=360$, eta $=0.05$, max_dept $=6$, subsample $=0.75)$ \\
\hline
\end{tabular}


Table S5. The architecture of the CNN regression model.

\begin{tabular}{|c|c|c|}
\hline Layer & Layer (type) & Output Shape \\
\hline 1 & Input layer & (None, $100,100,3)$ \\
\hline 2 & Convolution layer & (None, 98, 98, 32) \\
\hline 3 & Max pooling layer & (None, 49, 49, 32) \\
\hline 4 & Convolution layer & (None, $47,47,64$ ) \\
\hline 5 & Max pooling layer & (None, $23,23,64$ ) \\
\hline 6 & Convolution layer & (None, $21,21,128)$ \\
\hline 7 & Max pooling layer & (None, $10,10,128)$ \\
\hline 8 & Convolution layer & (None, $8,8,128$ ) \\
\hline 9 & Max pooling layer & (None, 4, 4, 128) \\
\hline 10 & Flatten layer & (None, 2048) \\
\hline 11 & Fully-connected layer & (None, 128) \\
\hline 12 & Dropout layer & (None, 128) \\
\hline 13 & Fully-connected layer & (None, 1) \\
\hline 14 & Output & (None, 1) \\
\hline
\end{tabular}


Table S6. The architecture of the MLP regression model.

\begin{tabular}{|c|c|c|}
\hline Layer & Layer (type) & Output Shape \\
\hline 1 & Input layer & (None, 18) \\
\hline 2 & Fully-connected layer & (None, 64) \\
\hline 3 & Fully-connected layer & (None, 256) \\
\hline 4 & Fully-connected layer & (None, 128) \\
\hline 5 & Dropout layer & (None, 64) \\
\hline 6 & Fully-connected layer & (None, 1) \\
\hline 7 & Output & (None, 1) \\
\hline
\end{tabular}


Table S7. Parameters of ML regression models.

\begin{tabular}{|c|l|}
\hline Algorithm & \multicolumn{1}{|c|}{ Parameters } \\
\hline Decision trees & $\begin{array}{l}\text { (criterion }=\text { 'mae', splitter }=\text { 'best', max_depth }=23 \text {, min_samples_leaf }=1, \\
\text { min_samples_split }=4)\end{array}$ \\
\hline $\begin{array}{c}\text { Random } \\
\text { forest }\end{array}$ & $\begin{array}{l}\text { (criterion }=\text { 'mse', n_estimators }=81, \text { max_depth }=23, \text { min_samples_leaf }=1, \\
\text { min_samples_split }=2)\end{array}$ \\
\hline SVM & (kernel = 'rbf', gamma $=1.26, \mathrm{C}=302)$ \\
\hline KNN & $\begin{array}{l}\text { (n_neighbors }=1, \text { weights }=\text { 'uniform', algorithm }=\text { 'auto', leaf_size }=30, \mathrm{p}=2, \\
\text { metric }=\text { 'minkowski') }\end{array}$ \\
\hline XGBoost & $($ num_round $=1000$, eta $=0.05$, max_dept $=7$, subsample $=0.5)$ \\
\hline
\end{tabular}


Table S8. Quantification performance for amikacin on the three datasets based on different regression models.

\begin{tabular}{|c|c|c|c|c|c|c|c|c|}
\hline & \multicolumn{8}{|c|}{ Training set } \\
\hline & $\begin{array}{l}\text { Linear } \\
\text { regression }\end{array}$ & DT & $\mathbf{R F}$ & SVM & KNN & XGBoost & MLP & CNN \\
\hline $\mathbf{R}^{2}$ & 0.640 & 0.998 & 0.995 & 1.000 & 1.000 & 1.000 & 0.996 & 0.999 \\
\hline MSE & 410.123 & 1.823 & 6.120 & 0.028 & 0.000 & 0.003 & 4.707 & 0.605 \\
\hline RMSE & 20.252 & 1.350 & 2.474 & 0.169 & 0.000 & 0.055 & 2.170 & 0.778 \\
\hline \multirow{3}{*}{ MAE } & 16.118 & 0.086 & 1.474 & 0.101 & 0.000 & 0.044 & 1.569 & 0.582 \\
\hline & \multicolumn{8}{|c|}{ Validation set } \\
\hline & $\begin{array}{l}\text { Linear } \\
\text { regression }\end{array}$ & DT & $\mathbf{R F}$ & SVM & KNN & XGBoost & MLP & CNN \\
\hline $\mathbf{R}^{2}$ & 0.630 & 0.973 & 0.965 & 0.994 & 0.998 & 0.984 & 0.994 & 0.999 \\
\hline MSE & 368.554 & 26.923 & 38.615 & 6.628 & 2.564 & 19.753 & 5.850 & 0.913 \\
\hline RMSE & 19.198 & 5.189 & 6.214 & 2.574 & 1.601 & 4.444 & 2.419 & 0.955 \\
\hline \multirow[t]{3}{*}{ MAE } & 15.551 & 1.346 & 4.144 & 0.900 & 0.128 & 2.195 & 1.839 & 0.662 \\
\hline & \multicolumn{8}{|c|}{ Test set } \\
\hline & $\begin{array}{l}\text { Linear } \\
\text { regression }\end{array}$ & DT & $\mathbf{R F}$ & SVM & KNN & XGBoost & MLP & CNN \\
\hline $\mathbf{R}^{2}$ & 0.590 & 0.891 & 0.963 & 0.989 & 0.996 & 0.983 & 0.991 & 0.997 \\
\hline MSE & 426.019 & 113.439 & 39.109 & 11.945 & 5.058 & 18.928 & 10.078 & 3.197 \\
\hline RMSE & 20.640 & 10.651 & 6.254 & 3.456 & 2.249 & 4.351 & 3.175 & 1.788 \\
\hline MAE & 16.243 & 2.977 & 3.919 & 1.441 & 0.318 & 2.335 & 2.044 & 1.040 \\
\hline
\end{tabular}

